\section{Idiopathic Thrombocytopenic Purpura (ITP) in the Elderly Over 75 Years: About a Rare Series in the Very Elderly Sub- jects}

\section{Abrar-Ahmad ZULFIQAR ${ }^{1^{*}}$, Amin TOUMI ${ }^{2}$, Nadir KADRI ${ }^{1}$, Jean DOUCET $^{1}$, Zoubir DJERADA ${ }^{3}$, Jean-Loup PENNAFORTE ${ }^{4}$ and Emmanuel ANDRES ${ }^{5}$}

${ }^{1}$ Department of Internal Medicine-Geriatrics-Therapeutics, University Hospital of Rouen, Rouen, France

${ }^{2}$ Emergency Unit, Hospital of Troyes, Troyes, France

${ }^{3}$ Toxicology and Pharmacovigilance Unit, University Hospital of Reims, Reims, France

${ }^{4}$ Department of Internal Medicine, University Hospital of Reims, Reims, France

${ }^{5}$ Department of Internal Medicine, University Hospital of Strasbourg, Strasbourg, France

\section{Abstract \\ Background \\ There is no specific data on the care of Idiopathic Thrombocyto- penic Purpura (ITP) of subjects aged 75 and over. \\ Methods}

Retrospective study between 2009 and 2015, focusing on subjects above age 75 , having been previously diagnosed with ITP in University Hospital of Reims.

\section{Results}

Fifteen patients were enrolled. The average age of diagnosis was 83.9 years, females representing 11 cases. The initial clinical presentation is dominated by hemorrhagic bleeding. During the diagnostic phase, the mean platelet count was $14,46 \mathrm{G} / \mathrm{I}$. The first line of treatment is based on oral corticosteroid therapy for 14 of the cases. At one month, a complete response for 9 patients and at 6 months,

*Corresponding author: Zulfiqar Abrar-Ahmad, Department of Internal Medicine-Geriatrics-Therapeutics, University Hospital of Rouen, Rouen, France, Tel: +33 0627102493; E-mail: abzulfiqar@gmail.com

Citation: Zulfiqar AA, Toumi A, Kadri N, Doucet J, Djerada D, et al. (2018) Idiopathic Thrombocytopenic Purpura (ITP) in the Elderly Over 75 Years: About a Rare Series in the Very Elderly Subjects. J Hematol Blood Transfus Disord $4: 017$

Received: August 07, 2017; Accepted: January 06, 2018; Published: January 22, 2018 recurrence for all patients. Splenectomy was performed for a single patient with recurrence after 6 months. Alternative treatments used include: mycophenolate mofetil, partial response for two patients and a failure for one, dapsone for 2 patients with partial responses, danazol for one patient with failed results, eltrombopag used for a single patient with adverse events that led to him stopping the medication. Rituximab had been used for 3 patients with a complete response for one patient and a partial response in the other two. Romiplostim was used for one patient with a complete response following the use of rituximab, which had provided a partial response for this patient. We incurred a loss of 5 patients. We noted one death within 1 month of the initial charge.

\section{Conclusion}

Large-scale studies should be conducted in the elderly, in order to standardize these practices.

Keywords: Corticosteroids; Elderly; ITP; Recurrence; Rituximab

\section{Introduction}

Idiopathic Thrombocytopenic Purpura (ITP), currently referred to as primary immune thrombocytopenic purpura, is an autoimmune disease linked to the presence of auto antibodies responsible for the peripheral destruction and central inhibition of platelet growth $[1,2]$. ITP is defined by an isolated thrombocytopenia less than $100 \mathrm{G} / \mathrm{L}$ in the absence of any other identified cause and/or associated disease [3]. The latter combines a highly variable clinical picture ranging from the absence of symptoms to mucocutaneous hemorrhaging (purpura, hematoma, epistaxis. . .) and even visceral (meningo-cerebral hemorrhaging), which can be a life-threatening prognosis [1]. ITP is a relatively rare disorder with an estimated incidence of approximately 5 new cases per 100,000 inhabitants per year in Europe [3]. Although it is usually found in children or young adults, predominantly female, ITP can occur at all stages of life, including the elderly (median age at diagnosis is close to 50 years of age). Therapeutic treatment of ITP is based primarily on the recommendations of experts and the analysis of patient cohorts. However, in 2016, there existed no such recommendations for the elderly. ITP can occur at all ages, but it is seen mainly in children and adult subjects, although rarely in the elderly over 75 years of age. At present, there is really no specific, precise, and documented data as to the treatment of subjects older than 75 years old affected by this pathology.

\section{Patients and Methods}

This is a retrospective study, upon consultation of medical records, centered on the collection of subjects over 75 years of age, having been diagnosed with ITP by the Internal Medicine Unit, University Hospital of Reims. The collection period ranges from 2009 to 2015. The demographic, clinical, treatment (effectiveness and side effects) and follow-up characteristics have been identified for each of the patients. The demographic, clinical and treatment characteristics (effectiveness and side effects, mainly at 1 and 6 months) as well as monitoring data were identified for all the patients.

The responses to the various treatments have been evaluated as follows: 
- Over $100 \cdot 10^{3}$ per cubic millimeter for a complete response.

- Over $50 \cdot 10^{3}$ per cubic millimeter with at least double the original figure of platelets for a partial response.

- An amount of platelets less than $50 \cdot 10^{3}$ per cubic millimeter constituted a failure.

The analysis of the data is made using an excel spreadsheet, which was anonymized. The declaration to CNIL is conducted by the DIM of the CHU Reims. The analysis of the data of the elderly over 75 years of age could not be compared with that of young subjects for more exact and meaningful statistical analysis due to a very low sample.

\section{Results}

\section{Patient characteristics}

15 patients were included during this period. The average age of diagnosis is 83.9 years of age, with a female predominance in 11 of the cases $(73.3 \%)$. The initial clinical presentation is dominated by hemorrhaging ( 2 episodes of hematuria, 1 episode of gingivorrhagia, 5 episodes of skin hemorrhaging of a purpuric nature, and an episode of melena), asthenia in 2 cases, and an asymptomatic characteristic in 5 cases.

In the medical histories of note, we noted the presence of autoimmune diseases preceding the diagnosis of ITP, including 1 case of autoimmune bullous dermatosis, a case of autoimmune hemolytic anemia, and a case of scleroderma. 2 malignant hematologic diseases (cutaneous lymphoma, LLC) were observed and a history of malignant tumor of the mammary in one patient. A follow-up for advanced dementia is ongoing for 2 patients. A diagnosis of systemic lupus was addressed simultaneously. Following the diagnosis of ITP, a vasculitis to ANCA was observed (in the follow-ups). In our series, we noted two patients on dabigatran and 3 patients on Acetylsalicylic acid $75 \mathrm{mg}$ per day.

\section{Treatment and responses}

As of the diagnosis of ITP, the first line of treatment was based on an oral corticosteroid in 14 cases. Bolus methylprednisolone was used (for the initial treatment as well as for recurrences) for 5 patients ranging from $250 \mathrm{mg}$ to $500 \mathrm{mg}$; the oral corticosteroid with a dosage of $1 \mathrm{mg} / \mathrm{kg}$ was used for 10 patients, followed by a gradual reduction. Regarding side effects, we note 1 case of steroid-induced diabetes. Regarding responses to corticosteroids, we note: in one month, a complete response for 9 patients, a partial response for 3 patients, and a failed response for 2 patients. At 6 months, a complete response for 0 patients and relapses for all patients. For the patients collected, the polyvalent immunoglobulins were not successful whether used alone or combined with corticosteroid therapy; as well as for the platelet transfusions used, without success, for 3 patients (transfusions conducted in association). The splenectomy was performed for only 1 patient with a relapse observed at 6 months and 1 year. The alternative treatments used were: mycophenolate mofetil for three cases with partial responses for 2 patients and a failed response for one patient (associated with undesirable effects including dysuria and gastric gravity), disulone for 2 patients with partial responses (with an adverse hepatic effect after 2 years from its introduction), danazol for one patient with failed response, eltrombopag used for a single patient with side effects that led to its cessation. Rituximab was used for 3 patients with a complete response for 1 patient and a partial response for 2 patients. Romiplostim was used for 1 patient with a complete response following the use of rituximab, which provided a partial response for this patient. We have 5 patients left to follow-up. We noted only one death within 1 month of initial treatment.

\section{Discussion}

We describe a very rare series based on ITP, occurring in the elderly patients over 75 years. There is no data described in the literature for the elderly patients over 75 years who are diagnosed ITP. The ITP treatment terms are based on the recommendations of experts, for which some are based on data and pediatric series [4]. To date, there are no specific recommendations with respect to those over 65 years of age affected by ITP. Previous studies were concerned with ITP affecting those over 60 years old, such as those of Linares et al. [5], those of Bizzoni et al., with the study of a cohort of 178 patients [6], and that of Daou S et al., which included a series of 47 patients with ITP, all over 60 years old [7]. Zulfiqar AA et al., have done work on ITP diagnosed in those over 65 years old, with 41 identified cases, with an average age of 76.5 years [8]. Our work focuses on subjects aged over 75 years old, for which an ITP diagnosis has been made, with an average age of diagnosis of 83.9 years. We have a female predominance in 11 cases of our series (73.3\%). Despite the absence of cerebral hemorrhagic signs in our series, it is to note that, in the literature, the clinical picture is more severe with respect to the pediatric and adult subjects (2); this is probably related to the weakness of the elderly subjects, polymedicated, with the consumption of drugs such as anticoagulants and/or antiplatelet agents. In terms of hematological data, the average platelet number in our series is $14.46 \mathrm{G} / \mathrm{L}$, which is lower compared to the study that we had conducted on ITP subjects over 65 years of age [8].

Analysis of our series shows that the treatment of our patients remains based, in the vast majority of cases, on the recommendations of experts not specific to seniors over 65 years old [9].

\section{Corticosteroid therapy}

Corticosteroid therapy, as illustrated in our series, remains the first line of treatment for all, including the elderly. Effectiveness of corticosteroid therapy, at 1 month (per os or preceded by a decision IV) remains satisfactory in our series, comparable to that of the literature [10].

In our series, there is however a net decrease of effectiveness of the corticosteroid therapy at 6 months. These results confirm a relatively low effectiveness of the corticosteroid therapy to induce a long-term remission. This criterion should therefore be taken into account in the treatment decision, particularly because of the frequent comorbidities and the weakness of these very elderly patients. In addition, corticosteroid therapy exposes this patient population to many secondary and non-negligible effects. Treatment with dexamethasone in high doses has been used in our series; in the literature, some authors have shown a greater effectiveness in acute situations, including the percentage of remissions over the long term [11].

\section{Polyvalent immunoglobulin}

In our series, polyvalent immunoglobulin alone has had no success, in the short and medium term, but a relative effectiveness in combination with corticosteroids, at one month, which is not the case 
at 6 months. We observed effectiveness in the very short term (in the order of 3 to 5 days), but a rapid decrease in the platelet count after 1 month. After these first lines of treatment, in case of relapse, there are some other available treatments.

\section{Splenectomy}

The splenectomy is considered to be one of the possible treatments, or even the treatment of reference in the second line of therapy [3]. We have just one single case of splenectomy, with a recurrence in 6 months. Conclusions cannot be drawn. Splenectomy has been studied by Kojouri et al., which showed, on a cohort of 2,623 adult patients, with a complete response rate of $66 \%$, and an $88 \%$ global response rate (average follow-up of 29 months) [12].

The risks of a splenectomy are not negligible, with a risk of infection, particularly among the frail elderly. In children and adult subjects, it remains the treatment of reference for ITP, but at a time of new alternative therapeutic healing (anti-CD20 antibodies) or suspensive (agonists of the Tpo-R) strategies, it is important to point out the existence of such infectious complications in order to prevent them (education, antibiotics, routine vaccinations). The indication for a splenectomy in the context of ITP for elderly subjects over 75 years of age must be discussed and reserved for severe arrays (platelets $<30 \mathrm{G} / \mathrm{L}$, presence of visceral hemorrhaging, associated with an anticoagulation and/or antiplatelet agent treatments). It is strongly recommended to wait for at least 1 year of evolution after the date of diagnosis of ITP before proposing the splenectomy given that healing of the ITP may occur up to this date. In all cases, it should rely on a multidisciplinary opinion and base it on a geriatric assessment, including comorbidities and the patient's prognosis (apart from the impact of the ITP) in its decisions.

\section{Disulone and dapsone}

Disulone and dapsone were used very little in our series. Danazol assures global remission over the long term in the context of ITP, as illustrated by the original work of Ahn et al., in a series of 96 patients (60 women and 36 men; 45 having had a splenectomy), having received danazol. In this cohort, the overall response rate to danazol is estimated to be $61.4 \%$. It is worth noting that the response is better in elderly females without a splenectomy - data confirmed by some authors [13]. Moreover, it should be underlined that the treatment comes with the occurrence of moderate hepatic cytolysis, which is the case in our series. Dapsone (sulfone family) was used for a single patient in our series and failed. It is also impossible to draw any conclusions. His interest in ITP was nevertheless confirmed and documented in several works, including that of Godeau et al., [14], which reports effectiveness in $30 \%$ of cases, with a good overall tolerance. The average daily dose of dapsone must be $75-100 \mathrm{mg} /$ day. It constitutes a phagocytic diversion and only has a suspensive effect. It is a good solution while waiting. It is necessary to guard against the occurrence of fatal DRESS syndromes.

\section{Biotherapy}

Concerning new therapies like the anti-CD20 antibody, such as rituximab, or the platelet growth factors, such as eltrombopag (administered orally), or romiplostim (administered subcutaneously), the data is more than incomplete as to their effectiveness and their tolerance in the elderly [2]. Rituximab (Mabthera ${ }^{\circledR}$, Roche) is a chimeric murine monoclonal antibody that binds specifically to the CD20 antigen expressed on the surface of the Lyb.
The administration of anti-CD20 causes thus a profound and prolonged Lyb depletion for 6 to 9 months but does not, in theory, cause hypogammaglobulinemia due to the preservation of the Lyb memory pool, which could explain the tolerance to the treatment, especially in terms of risk of infection. In adults, a retrospective study based on 300 patients showed that $60 \%$ of patients having ITP, splenectomized or not, respond to treatment, with an extended response observed in $40 \%$ of cases [15]. An open prospective study and 2 randomized studies have documented its effectiveness [16,17]. Thus, the response rate is estimated at about $40 \%$ at 1 year, with a sustained response in approximately $20 \%$, which may lead to healing. It is noted that $90 \%$ of the responses are observed in 8-10 weeks after the initiation of treatment. Rituximab is currently used as a third line of treatment in case of failure/contraindication of the splenectomy [3].

This indication is the subject of a temporary treatment protocol in France. The question of the dosage is not resolved. For adults, the posology of 2 injections of $1000 \mathrm{mg}$ at 2-week intervals would be as effective as the "lymphoma treatment" (4 injections of $375 \mathrm{mg} / \mathrm{m}^{2}$ ). All of our patients have been treated in this way. In terms of tolerance and safety of the product, a prospective registry was set up by the Reference center for adults autoimmune cytopenia (Pr Godeau, Henri-Mondor, Hospital AP-HP, Paris). Its follow-up over a period of 5 years will help to better assess the tolerance of the product.

The risk of multiprogressive leukoencephalopathy remains very exceptional. Thus, in light of this data, it seems legitimate to discuss the place of rituximab as a second line of treatment at the same level as the splenectomy. There are more studies regarding adult subjects concerning rituximab. Numerous tests showed the interest of rituximab in this situation with effectiveness in the short term but frequent relapses over the long term. Thus, a study was conducted to understand the mechanisms of action of rituximab during ITP. This study showed that treatment with rituximab has more chance to be effective if it is administered early in the course of ITP in a young patient [18]. This study thus confirms the work of Stasi et al., who have shown that rituximab in addition to induce an extended B lymphocyte depletion also impacted the repertoire of $\mathrm{T}$ cells. They showed that throughout IPT, rituximab is able to restore pre-existing abnormalities affecting the LyT, among which: the Th2/Th1 and Tc1/Tc2 balance, but also the proportion of LyT regulators that could account for the prolonged successes observed in some patients. Thus, a long evolution of ITP would be associated with an oligoclonal T lymphocyte expansion, which could be one of the resistance factors to rituximab [19]. The results of Viallard JF et al., in the French prospective registry PTI-RITUX [18], also shows for the first time that a complete response to corticosteroids administered in the first line of treatment is a factor of a good long-term response for the administration of rituximab at a later stage.

The thrombopoietin receptor agonists have the AMM in adults with chronic ITP refractory to other treatments in the event of failure/ contraindication of the splenectomy. The two-platelet growth factors showed overall efficacy rates in the order of $70 \%$ in the short term and over $50 \%$ of extended responses [20]. The tolerance profile remains good in the short and medium term. There is nevertheless an important risk of thrombosis in elderly subjects, which is the case in our series, for one of our patients (where prudent use in patients with thrombotic history or risk of thrombosis), and inducement of myelofibrosis (reassuring recent data). Their cost remains particularly high and one of the limits to their use remains their suspensive effect 
(decline in the rate of platelets occurring immediately after their cessation).

\section{Limitations of the Study}

The retrospective nature and small size make the interpretation of the results and their generalization difficult or even questionable. Moreover, some patients of our series have been lost to follow-up, where the study of the evolution over the long-term could not be completed.

\section{Conclusion}

Studies on ITP in the very elderly are very rare and their treatment remains poorly covered in scientific literature. Our work reports on a very rare series, focusing on ITP subjects diagnosed beyond 75 years, and relays interesting data about their treatment. The first line of treatment of newly diagnosed ITP is now well-codified. This is not the case for the second line of treatment and the respective place of the different treatments, including the splenectomy, rituximab and Thrombopoietin (TPO) agonists, which remain a matter of debate, in children and adult subjects, and by extension in the elderly, and depending on their degree of autonomy and frailty, their comorbidities must be taken into account prior to their establishment.

\section{Declaration}

Authors declare any financial supports or relationships.

\section{Conflict of Interest}

"No potential conflicts of interest were disclosed".

\section{Reference}

1. McMillan R (2000) The pathogenesis of chronic immune (idiopathic) thrombocytopenic purpura. Semin Hematol 37: 5-9.

2. Andrès E, Federici L, Zimmer J, Daou S, Ciobanu E, et al. (2008) Traitement du purpura thrombopénique idiopathique du sujet âgé. Med Ther $14: 25-31$.

3. Purpura thrombopénique immunologique de l'enfant et de l'adulte (2009) Protocole National de Diagnostic et de Soins, Haute Autorité de santé, France.

4. Cines DB, Blanchette VS (2002) Immune thrombocytopenic purpura. $\mathrm{N}$ Engl J Med 346: 995-1008.

5. Linares M, Cervero A, Colombina P, Pastor E, Lopez A, et al. (1995) Chronic idiopathic thrombocytopenic purpura in the elderly. Acta Haematol 93: $80-82$.

6. Bizzoni L, Mazzucconi MG, Gentile M, Santoro C, Bernasconi S, et al. (2006) Idiopathic Thrombocytopenic Purpura (ITP) in the elderly: clinical course in 178 patients. Eur J Haematol 76: 210-216.

7. Daou S, Federici L, Zimmer J, Maloisel F, Serraj K, et al. (2008) Idiopathic thrombocytopenic purpura in elderly patients. A study of 47 cases from a single reference center. Eur J Intern Med 19: 447-451.
8. Zulfiqar AA, Novella JL, Mahmoudi R, Pennaforte JL, Andres E (2016) Treatment in idiopathic thrombocytopenic purpura in the elderly: About a retrospective study. Geriatr Psychol Neuropsychiatr Vieil 14: 151-157.

9. British Committee for Standards in Haematology General Haematology Task Force (2013) Guidelines for the investigation and management of idiopathic thrombocytopenic purpura in adults, children and in pregnancy. Br J Haematol 120 : 574-96.

10. Leung AY, Chim CS, Kwong YL, Lie AK, Au WY, et al. (2001) Clinicopathologic and prognostic features of chronic idiopathic thrombocytopenic purpura in adult Chinese patients: An analyse of 220 cases. Ann Hematol 80: $384-386$.

11. Borst F, Keuning JJ, van Hulsteijn H, Sinnige H, Vreugdenhil G (2004) High-dose dexamethasone as first- and second-line treatment of idiopathic thrombocytopenic purpura in adults. Ann Hematol 83: 764-768.

12. Kojouri K, Vesely SK, Terrel DR, George JN (2004) Splenectomy for adults with idiopathic thrombocytopenic purpura: A systematic review to assess long-term platelet count responses, prediction of response, and surgical complications. Blood 104: 2623-2634.

13. Ahn YS, Rocha R, Mylvaganam R, Garcia R, Duncan R, et al. (1989) Long-term danazol therapy in autoimmune thrombocytopenia : Unmaintained remission and age-dependent response in women. Ann Intern Med 111: 723-729.

14. Godeau B, Durand JM, Roudot-Thoraval F, Tennezé A, Oksenhendler E, et al. (1997) Dapsone for chronic autoimmune thrombocytopenic purpura: a report of 66 cases. Br J Haematol 97: 336-339.

15. Arnold DM, Dentali F, Crowther MA, Meyer RM, Cook RJ, et al. (2007) Systematic review: efficacy and safety of rituximab for adults with idiopathic thrombocytopenic purpura. Ann Intern Med 146: 25-33.

16. Godeau B, Porcher R, Fain O, Lefrère F, Fenaux P, et al. (2008) Rituximab efficacy and safety in adult splenectomy candidates with chronic immune thrombocytopenic purpura: results of a prospective multicenter phase 2 study. Blood 112: 999-1004.

17. Zaja F, Baccarani M, Mazza P, Bocchia M, Gugliotta L, et al. (2010) Dexamethasone plus rituximab yields higher sustained response rates than dexamethasone monotherapy in adults with primary immune thrombocytopenia. Blood 115: 2755-2762.

18. Viallard JF, Khellaf M, Charles-Nelson A, Fain O, Hamidou M, et al. (2014) Peut-on prédire la réponse à long terme au rituximab au cours du purpura thrombopénique immunologique? Résultats après un an de suivi chez 255 patients inclus dans le registre prospectif français PTI-RITUX. La Revue de médecine interne 35.

19. Stasi R, Del Poeta G, Stipa E, Evangelista ML, Trawinska MM, et al. (2007) Response to B-cell depleting therapy with rituximab reverts the abnormalities of T-cell subsets in patients with idiopathic thrombocytopenic purpura. Blood 110: 2924-2930.

20. Cheng G, Saleh MN, Marcher C, Vasey S, Mayer B, et al. (2011) Eltrombopag for management of chronic immune thrombocytopenia (RAISE): A 6-month, randomised, phase 3 study. Lancet 377: 393-402. 